\title{
PULMONARY PERFUSION SCINTIGRAPHY AND SURVIVAL IN LUNG CARCINOMA PATIENTS TREATED WITH CHEMOTHERAPY AND RADIOTHERAPY
}

\author{
N. Georgieva ${ }^{1 *}$, E. Obretenov ${ }^{2}$, G. Kalaydjiev ${ }^{3}$, P. Chilingirov ${ }^{4}$, K. Peeva ${ }^{5}$ \\ ${ }^{1}$ Department of Nuclear Medicine, Medical Faculty, Trakia University, Stara Zagora, Bulgaria \\ ${ }^{2}$ Clinic of Thoracic Surgery, UMBAL, Stara Zagora, Bulgaria \\ ${ }^{3}$ Clinic of Thoracic Surgery SBALO, Sofia, Bulgaria \\ ${ }^{4}$ Oncology Center, Stara Zagora, Bulgaria \\ ${ }^{5}$ Department of Social Medicine and Health Management, Medical Faculty, Trakia University, \\ Stara Zagora, Bulgaria
}

\begin{abstract}
Purpose: The best treatment for lung cancer is a surgical resection. Patients in advanced stage are subject to chemotherapy and/or radiotherapy. Our aim is to evaluate whether the survival is related to perfusion index, stage and presence of radio and/or chemotherapy.

Materials And Methods: We analyzed the follow-up of 125 patients (118 men and 7 women, 30-76 years of age) with lung perfusion scintigraphy made between years 1989 and 1998 until 2013. Fortythree patients have been treated by radio-chemotherapy and 82 by chemotherapy. Patients have been staged by TNM classification from II to IV clinical stage. Survival was calculated by the KaplanMeier method, and groups were compared by the Mantel-Cox Log Rank test.

Results: Perfusion changes varied from impaired in different extent perfusion to lack of perfusion in one of the lungs. The Median survival of radio-chemotherapy treated patients with perfusion index of affected lung less than $49 \%$ was 10,5 months and with perfusion index greater than $49 \%$ was 18,4 months. The overall Median survival of radio-chemotherapy treated patients was 11,2 months. The median survival of chemotherapy treated patients with perfusion index of affected lung less than $49 \%$ was 7,9 months and with perfusion index greater than $49 \%$ was 4,4 months. The overall Median survival for chemotherapy treated patients was 6,9 months.

Conclusion: Perfusion index and stage were not statistically significant factors for the survival of patients with radio-chemotherapy and chemotherapy. Overall radio-chemotherapy treated patients have better median survival than the median survival by stage
\end{abstract}

Key words: lung carcinoma, lung perfusion scintigraphy, chemotherapy, radiotherapy, survival

The best treatment for lung cancer is surgical resection. Patients in advanced stage are subject to chemotherapy and/or radiotherapy.

\section{MATERIALS AND METHODS}

We analyzed retrospectively 125 consecutive cases with primary lung carcinoma treated in our departments. Every patient was subject to a standard postero-anterior and lateral chest Xray, fiberoptic bronchoscopy and to some of them had chest CT and video-assisted thoracoscopy (VATS). We studied 118 men and 7 women, between 30 and 76 years old.

\footnotetext{
*Correspondence to: Nina Georgieva, Department of Nuclear Medicine, Medical Faculty, 11 Armeyska str. ,6003 Stara Zagora, Bulgaria, Email:ngeorgieva21@yahoo.com
}

The tumor was located in the right lung in 82 patients and in the left lung in the remaining 43 cases.

Lung perfusion scintigraphy was performed in all patients with $99 \mathrm{~m}$ Tc- human albumin microspheres with a particle size of $23-45 \mu \mathrm{m}$ (kit Sferotec - Sorin Biomedica-Italy). The injection was applied in supine position, intravenously in bolus, $1 \mathrm{ml}$ at an activity of 55-74 MBq. The period of examination was between the $15^{\text {th }}$ min and the $2^{\text {nd }}$ hour after injection, at anterior, posterior, lateral and $45^{\circ}$ detection, perfusion indices from 6 fields in front and posterior projection were calculated (Picker Dyna Camera 4 Scintillation Camera System - Picker Corporation Nuclear and Ultrasound, USA). 
The patients treated with radio-chemotherapy were 43: 42 men and 1 woman, age range from 46 to 70 years. Treated only with radiotherapy were 3 patients. Treated only with chemotherapy were 82 patients -76 men and 6 women, age range from 30 to 76 years. The patients were staged in accordance with TNM classification (1). From the patients with radiochemotherapy in II stage were 3 patients, in IIIA stage were 18 patients, in IIIB stage were 17 patients and in IV stage were 4 patients. The radiotherapy was telegamma therapy fractionated by 2 Gy daily, 5 days a week exposure to reach a total dose of $50 \mathrm{~Gy}$ in 10 of the patients (in 4 patients - 50 Gy, in 4-40 Gy, in 1-60 Gy and in 1-30 Gy). All other patients were treated in schemes of high-dose fractionated telegamma therapy, equivalent to $50 \mathrm{~Gy}$ (courses and combinations most often of $5 \times 4$ Gy, 4x5 Gy, 5x3 Gy and others). The chemotherapy treated patients were distributed in the following stages: II stage -1 patient, IIIA stage -34 patients, IIIB stage -15 patients and in IV stage -31 patients. The chemotherapy patients were treated with the following combinations: Vincristine, Cyclophosphamide, Methotrexate (VCM) or Mitomycin, Ifosfamide, or Navelbine (Vinorelbine), Cisplatin. The lung carcinoma developed in 2 patients after previous tuberculosis. In 3 of the patients the lung carcinoma was a second tumor after bladder carcinoma, ear carcinoma or carcinoma of the colon. In 1 patient it was $3^{\text {rd }}$ tumor after tongue carcinoma and parotid glandule.

As beginning of the disease, we considered the date of the registration of the patients in the Oncology center. The patients were examined during the period 1989 to 1999 , and were observed for survival until September, 2013. For some of the patients the clinical stage was changed during the follow-up. Survival analysis was performed by the Kaplan-Meier method. Wilcoxon rank sum test was used to compare groups by stage and perfusion index (less than 49\% and higher than 49\%). The test for comparing the equality of the survival distributions between the study groups was done by use of the Mantel-Cox Log Rank, with an inclusion criterion of a $\mathrm{P}$ value less than 0,05. All statistical analysis were performed with the IBM SPSS Statistics 19 software.

\section{RESULTS}

The lung perfusion scintigraphy demonstrated an enlarged mediastinum in front and posterior at 46 of the patients treated with chemotherapy and radio-chemotherapy. In some cases the mediastinum is shifted. A zone with polycyclic borders and decreased perfusion was present in 51 patients. Impaired perfusion in both hili was found in 6 patients and in 1 patient the data were uncertain. There was one patient with impaired perfusion of the hilum and in the other hilum a perfusion defect was found. Perfusion changes in the homo-lateral hilum were found in 35 patients. The perfusion changes in the affected lung encompass different extents of impaired perfusion: to perfusion defect in at least three segments; to lack of perfusion in at least one lobe or a whole lung. A concentrically shrunk image of the affected lung was detected in part of the patients with main bronchus tumor. On Figure $\mathbf{1}$ is presented the pulmonary perfusion scintigraphy of 72-year-old patient with final diagnosis Carcinoma of the left main bronchus T3N2Mx, III stage, with histology: differentiated spinous-cellular carcinoma; from the fibro-bronchoscopy the bronchus was stenotic by the tumor formation in $3 \mathrm{~cm}$ from the bifurcation, the treatment was chemotherapy. The patient survived 13 months.

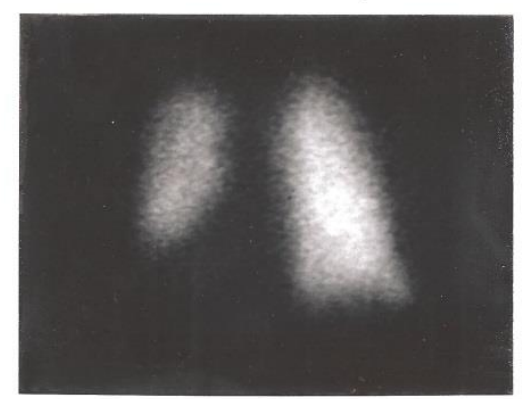

Figure 1. M.72years. Perfusion scintigraphy in front and posterior projections: left lung is with concentrically shrunk image, more distinct in posterior projection, with impaired perfusion in the periphery in both projections, and in the hilum in posterior projection. There is a zone of impaired perfusion medially in right apex. Perfusion indices: right lung $65 \%$, left lung $35 \%$.

In part of the patients with main bronchus tumor the perfusion was severely impaired or was lacking in the whole lung. On Figure 2 is presented the perfusion scintigraphy of 54-year old patient with final diagnosis Left lung carcinoma T3N3Mx, III stage. The 
bronchoscopy detects that the left main bronchus is stenotic and infiltrated by the tumor formation. On the perfusion scintigraphy in front and posterior projections lacked

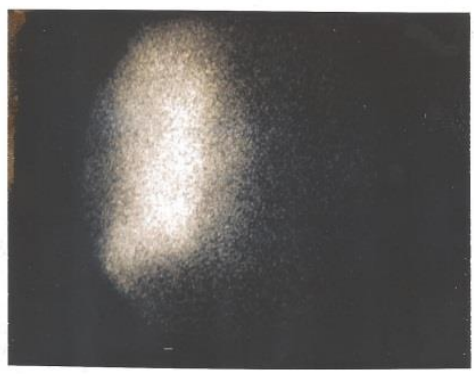

GEORGIEVA N., et al. perfusion in left lung, while in the right hilum the perfusion is impaired in both projections. The treatment was chemotherapy, the patient survived 6 months.

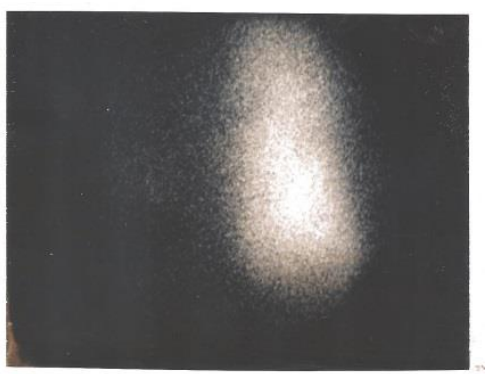

Figure 2. Perfusion scintigraphy in front and posterior projections of a 54 year old patient with left main bronchus carcinoma - lack of perfusion in left lung, and in the hilum of the right lung it is embarrassed in both projections.

There were bilateral perfusion changes in 25 patients. Bilateral perfusion changes were found in one patient with a tracheal section carcinoma. On Figure $\mathbf{3}$ is presented the pulmonary perfusion scintigraphy in front and posterior projections of 68-year old patient with final diagnosis Right bronchus carcinoma, III clinical stage. The bronchoscopy detects that the lumen of the trachea in its distal end is almost entirely occluded from a big tumor formation. On the pulmonary perfusion scintigraphy the mediastinum is shifted on the right in both projections. In front projection -

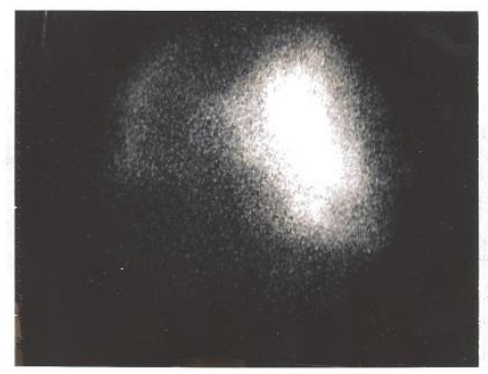

insignificant to lacking perfusion of the right lung, the left hilum was pulled towards the mediastinum and in 8 and 9 segments of the left lung and at its periphery the perfusion was impaired. In posterior projection - severely impaired perfusion in the right lung with totally decreased perfusion image and impaired perfusion in the left lung periphery. The perfusion indices were: right lung 20\%, left lung - $80 \%$. Chemotherapy and telegamma therapy were applied to the patient (by scheme $5 \times 3$ Gy).

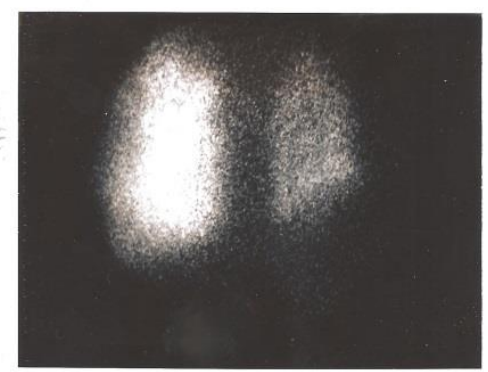

Figure 3. Pulmonary perfusion scintigraphy in front and posterior projections of 68 year old patient with tracheal carcinoma.

In the patients with apical carcinoma type Pancoast-Tobias was found strongly impaired perfusion or apical defect in part of the 1,2 and 3 segments, with curved border towards the lung, in some cases accompanied by enlarged in its cranial half mediastinum. On

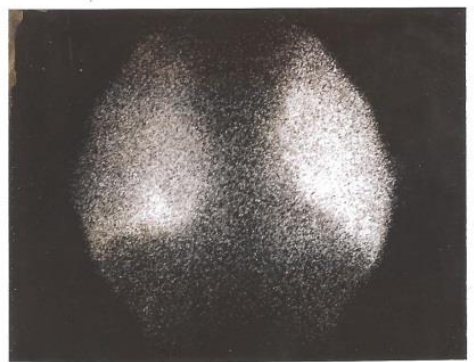

Figure 4 is shown the pulmonary perfusion scintigraphy in front and posterior projections of 57-year old patient with final diagnosis Carcinoma of the upper lobe of the right lung VCS syndrome before treatment.

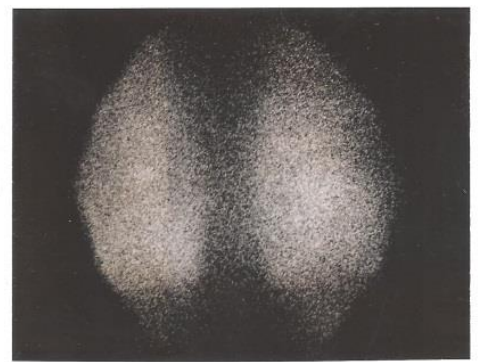

Figure 4. Perfusion scintigraphy in front and posterior projections of a 57 year old patient with moderate to severe embarrassed perfusion in 1,2 and 3 segments of the right lung, lightly impaired perfusion in both hili and enlarged in cranial third mediastinum. 
The pulmonary perfusion scintigraphy of the same patient 5 months later is shown on Figure 5. He was treated by chemotherapy -3 courses according to scheme VCM and telegamma therapy $2 \mathrm{x} 8,5 \mathrm{~Gy}=35 \mathrm{~Gy}$

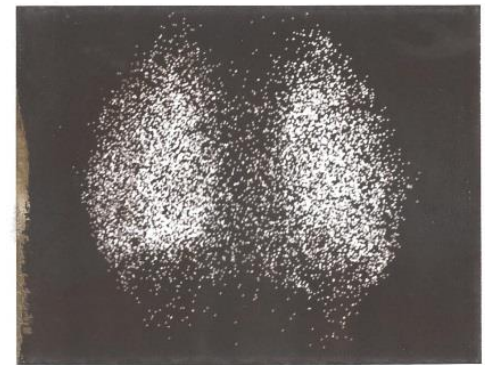

GEORGIEVA N., et al. supraclavicular and $5 \mathrm{x} 4 \mathrm{~Gy}=32 \mathrm{~Gy}$, right sternal and scapular. He survived 7 months.

Pulmonary perfusion scintigraphy was applied before and after treatment in part of the patients treated by chemotherapy.

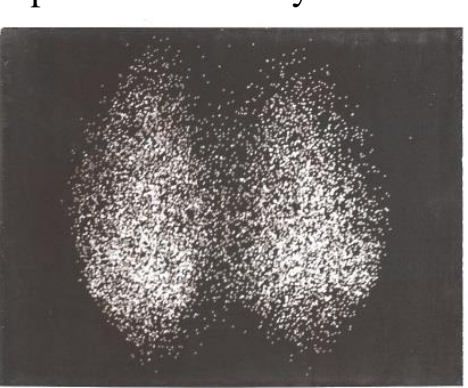

Figure 5. Perfusion scintigraphy in front and posterior projections of the patient from Figure $4-5$ months after treatment. In comparison with previous perfusion scintigraphy progression of perfusion changes is not observed.
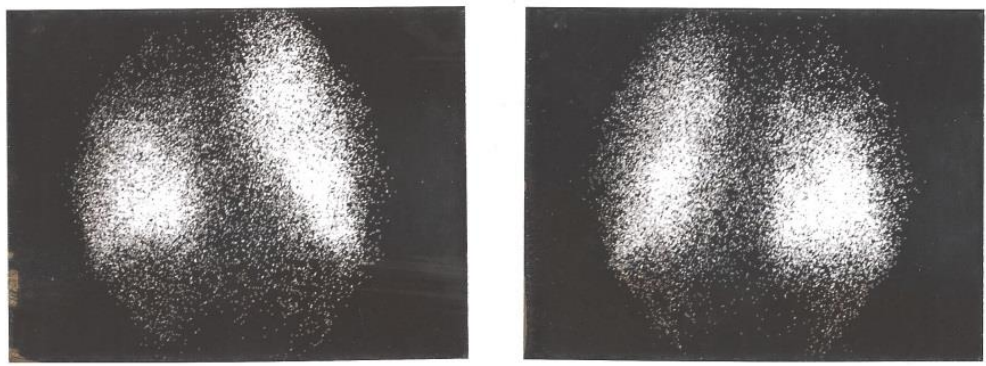

Figure 6. Perfusion scintigraphy in front and posterior projections of patient $M-62$ years old before chemotherapy. Perfusion defect in the region of 1,2 and 3 segments of right lung with embarrassed perfusion in left hilum and in left apically in posterior projection and enlarged in posterior projection mediastinum.

On Figure 6 the is presented pulmonary perfusion scintigraphy of 62 years old patient before the start with chemotherapy. The diagnosis is Carcinoma of right bronchi, III stage, with bronchoscopy: tumor originating from the lateral wall of right main bronchus, encompassing the entrance of the upper lobe bronchus. He survived 14 months. On Figure 7 is presented perfusion scintigraphy of the same patient after 11months and chemotherapy.
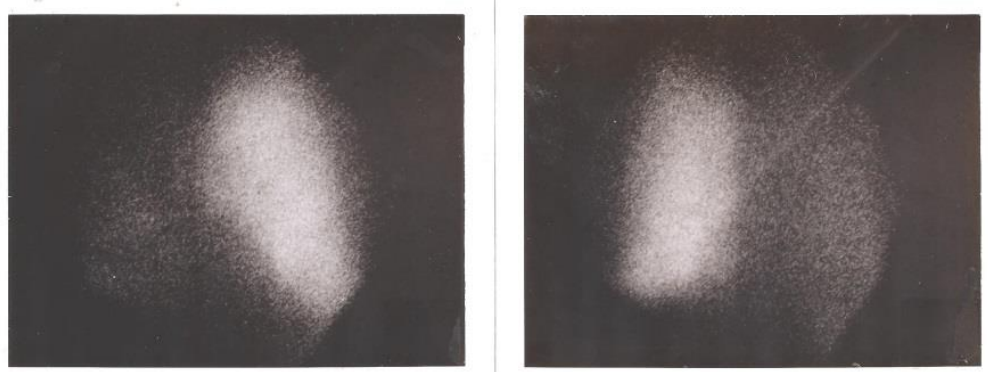

Figure 7. Pulmonary perfusion scintigraphy of the patient from Figure 6 in front and posterior projections. Lacking to insignificant perfusion in right lung and embarrassed perfusion apically and in zone of polycyclic borders on projection place of left hilum, clearly distinct in posterior projection.

In patients treated by radio-chemotherapy perfusion indices at localization in the right lung had Mean $=40,37 \%, \quad \mathrm{SD}=12,32 \%$, $\mathrm{SE}=2,56 \%$, and $95 \%$ CI $(35,04 ; 45,70)$. Perfusion indices at localization in the left lung (14 of the patients) were Mean $=29,98 \%$, $\mathrm{SD}=8,97 \%, \mathrm{SE}=2,99 \%$, and $95 \% \mathrm{CI}(23,08 \%$; $36,88 \%)$. Perfusion indices at affected lung, independently of right or left lung, had Mean $=37,45 \%, \mathrm{SD}=12,29 \%, \mathrm{SE}=2,17 \%$, and $95 \%$ CI $(33,02 \% ; 41,88 \%)$.
As a cutoff point between small and significant perfusion impairments we have defined $49 \%$, close to the normal ratio of perfusion indices right-left. In these two groups we have searched for relation between perfusion indices and survival of the patients.

The survival of patients treated with radiochemotherapy with Perfusion index of the right lung less than $49 \%$ had Mean=23,93 months with $\mathrm{SE}=6,44$ and $95 \%$ CI $(11,30 ; 36,56)$; Median=10,50 months with $\mathrm{SE}=1,50$ and $95 \%$ 
CI $(7,56 ; 13,44)$. The survival of patients with Perfusion index of the right lung more than $49 \%$ had Mean=83,14 months with $\mathrm{SE}=42,32$ and $95 \% \mathrm{CI}(0,20 ; 166,08)$; Median $=18,43$ months with SE=30,29 and 95\% CI $(0 ; 77,81)$.
GEORGIEVA N., et al. Total survival of the patients with affected right lung had Mean=33,31 months with $\mathrm{SE}=9,06$ and $95 \% \quad \mathrm{CI} \quad(15,56 ; \quad 51,06)$; Median $=11,20$ months with $\mathrm{SE}=1,8$ and $95 \%$ CI $(7,68 ; 14,72)$. (Figure 8)

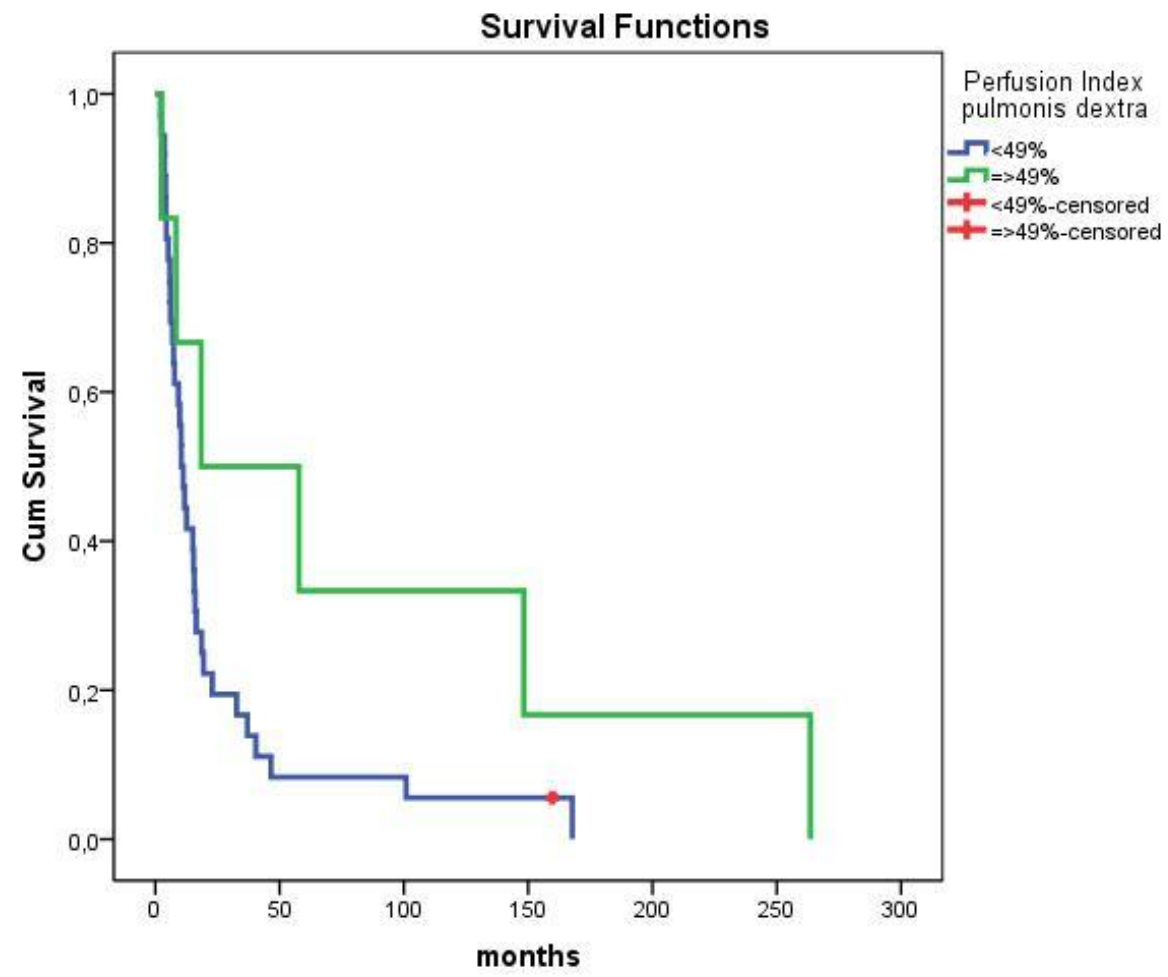

Figure 8. Survival of the radio-chemotherapy patients in months with perfusion index under and over $49 \%$ with affected right lung.

All patients treated with radio-chemotherapy with affected left lung were with perfusion less than $49 \%$, that is why their total survival had
Mean $=33,31$ months with $\mathrm{SE}=9,06$ and $95 \%$ CI $(15,56 ; 51,06)$; Median=11,20 months with $\mathrm{SE}=1,79$ and $95 \% \mathrm{CI}(7,68 ; 14,72)$ (Figure 9).

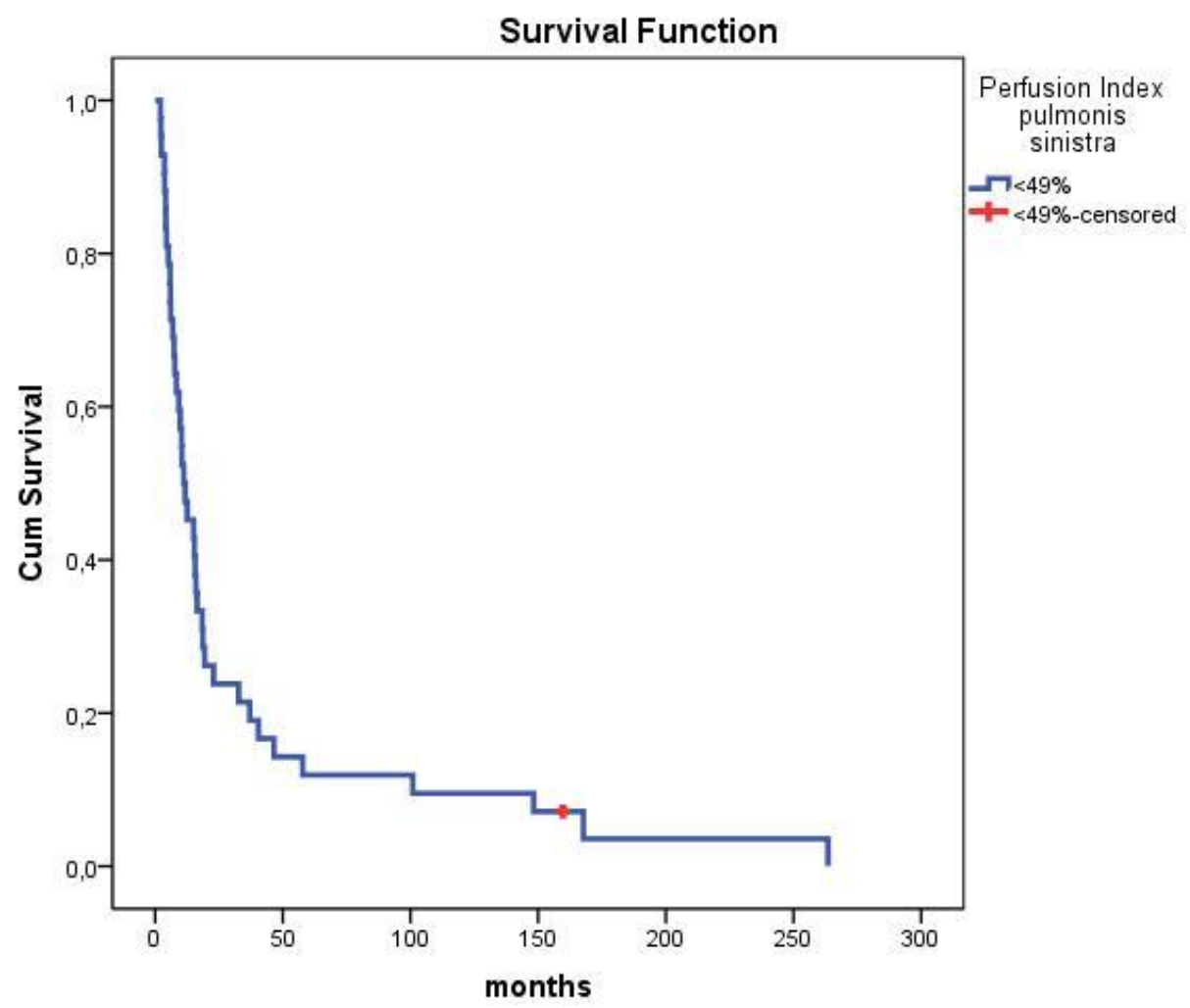

Figure 9. Survival of the radio-chemotherapy patients in months with perfusion index under $49 \%$ with affected left lung. 
Survival of the patients treated with radiochemotherapy with perfusion index of affected lung, right or left independently, less than $49 \%$ had Mean=23,93 months with $\mathrm{SE}=6,44$ and 95\% CI $(11,30 ; 36,56)$; Median=10,50 months with $\mathrm{SE}=1,50$ months and 95\% CI (7,56; $13,44)$. Survival of the patients with perfusion index of affected lung more than $49 \%$ had
GEORGIEVA N., et al. Mean=83,14 months with $\mathrm{SE}=42,32$ months and $95 \%$ CI $(0,20 ; 166,08)$; Median $=18,43$ months with SE=30,29 and 95\% CI $(0 ; 77,81)$. Total survival of the patients with affected right or left lung had Mean=33,31 months with $\mathrm{SE}=9,06$ and $95 \%$ CI $(15,56 ; 51,06)$; Median=11,20 months with $\mathrm{SE}=1,8$ and $95 \%$ CI $(7,68 ; 14,72)$ (Figure 10).

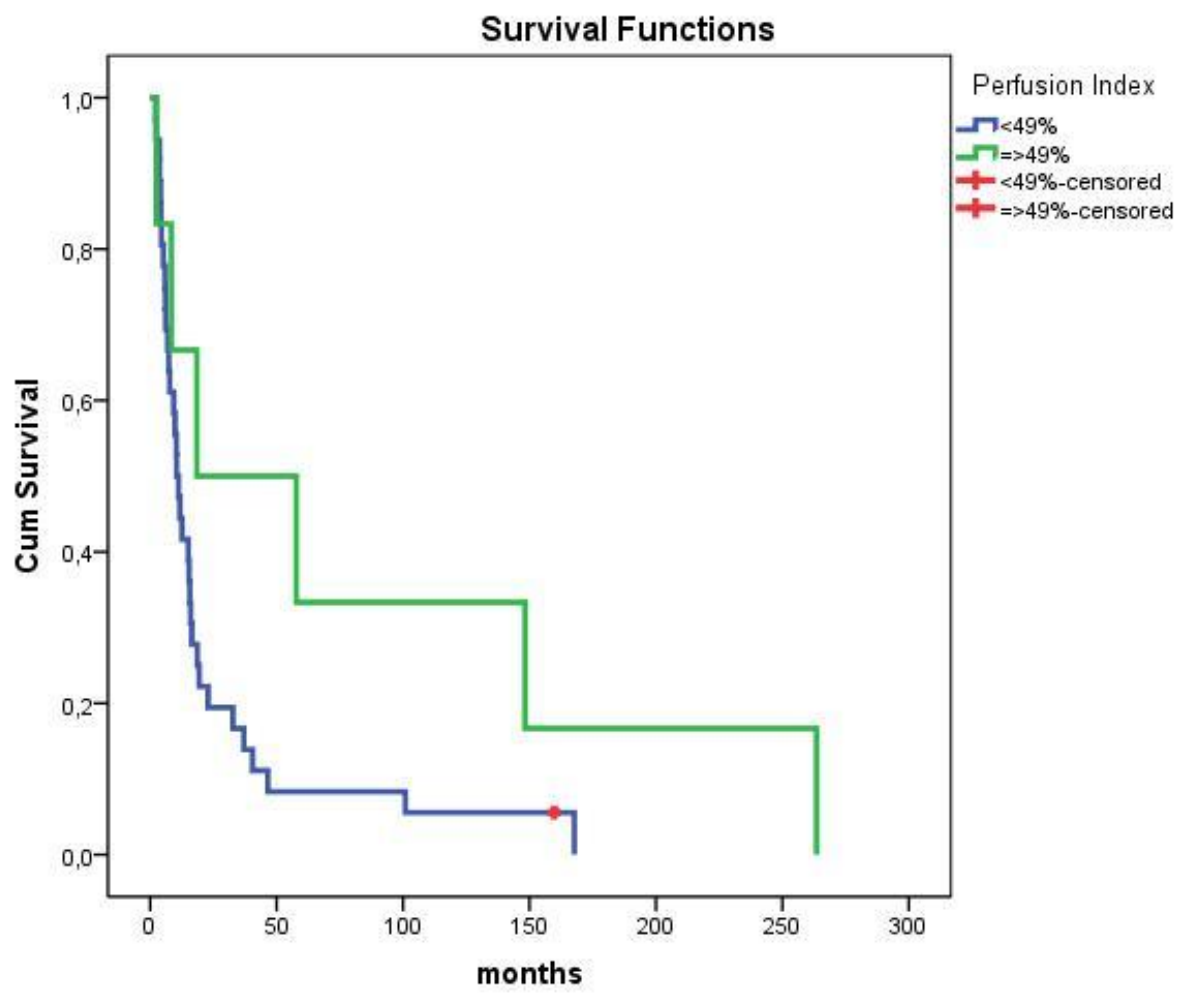

Figure 10. Survival of the radio-chemotherapy patients in months with perfusion index under and over $49 \%$ independent of the affected lung.

There exists a tendency for better median of survival of the patients treated with radiochemotherapy at small perfusion impairments, but we found the perfusion index not to be statistically significant factor for survival of the patients.

In one of the groups there was only one patient who survived. In the group with perfusion index of the right lung more than 49\% were 6 patients, all of them passed away, three have survived up to 18,4 months, one has survived 148 months and one 263,5 months. While from the 36 patients with perfusion index less than $49 \%$ half of them have survived 10,5 months.

Survival of the patients by stages in radiochemotherapy for II stage had Mean $=36,12$ months with $\mathrm{SE}=32,39$ and $95 \%$ CI $(0,8$; 99,60); Median=3,77 months with $\mathrm{SE}=0,06$ and $95 \%$ CI $(3,66 ; 3,88)$. For III A stage: Mean $=36,54$ months with $\mathrm{SE}=15,61$ and $95 \%$ CI $(5,94 ; 67,13)$; Median=10,4 months with $\mathrm{SE}=3,47$ and $95 \%$ CI $(3,6 ; 17,2)$. For III $\mathrm{B}$ stage: Mean $=33,36$ months with $\mathrm{SE}=12,65$ and $95 \%$ CI $(8,57 ; 58,14)$; Median=16 months with $\mathrm{SE}=4,1$ and $95 \%$ CI $(7,97 ; 24,03)$. For IV stage: Mean $=10,98$ months with $\mathrm{SE}=1,77$ and 95\% CI $(7,51 ; 14,45)$; Median $=9,83$ months with $\mathrm{SE}=2,42$ and $95 \%$ CI $(5,08 ; 14,57)$. The total survival of the patients stage independent had Mean $=33,92$ months with $\mathrm{SE}=9,25$ and 95\% CI $(15,79 ; 52,06)$; Median $=11,20$ months with $\mathrm{SE}=2,85$ and $95 \%$ CI $(5,62 ; 16,27)$ (Figure 11). The stage does not appear to be statistically significant factor for survival of the patients $(\mathrm{P}=0,877)$. The small number of cases in stage II (3 patients) and in stage IV (4 patients), as well as only one survived patient probably were the reasons for that.

In patients treated only with chemotherapy, the perfusion indices at localization of the process in the right lung had Mean $=46,78 \%$, $\mathrm{SD}=11,43 \%, \mathrm{SE}=2,16 \%$, and $95 \% \mathrm{CI}(42,35 \%$; $51,21 \%)$. The perfusion indices of the affected lung had Mean=36,92\%, $\mathrm{SD}=9,62 \%$, $\mathrm{SE}=2,1 \%$, and $95 \% \mathrm{CI}(32,54 \% ; 41,3 \%)$. The perfusion indices of the affected lung no matter left or right had Mean $=42,56 \%, \mathrm{SD}=11,68 \%$, $\mathrm{SE}=1,67 \%$, and $95 \%$ CI $(39,2 \% ; 45,91 \%)$. Survival of the patients with perfusion index of the right lung less than $49 \%$ had Mean=26,6 months with $\mathrm{SE}=7,53$ and $95 \%$ CI $(11,84$; 
41,36); Median=7,93 months with $\mathrm{SE}=2,3$ and $95 \%$ CI $(3,42 ; 12,44)$. Survival of the patients with perfusion index of the right lung more than $49 \%$ had Mean=22,23 months with $\mathrm{SE}=14,97 \quad$ and $95 \% \quad \mathrm{CI} \quad(0 ; \quad 51,58)$; Median=4,47 months with $\mathrm{SE}=2,95$ and $95 \%$
GEORGIEVA N., et al. CI $(0 ; 10,16)$. The total survival of the patients with affected right lung had Mean=25,56 months with $\mathrm{SE}=6,89$ and $95 \%$ CI $(12,06$; $39,07)$; Median=7,6 months with $\mathrm{SE}=1,48$ and 95\% CI (4,69; 10,51) (Figure 12).

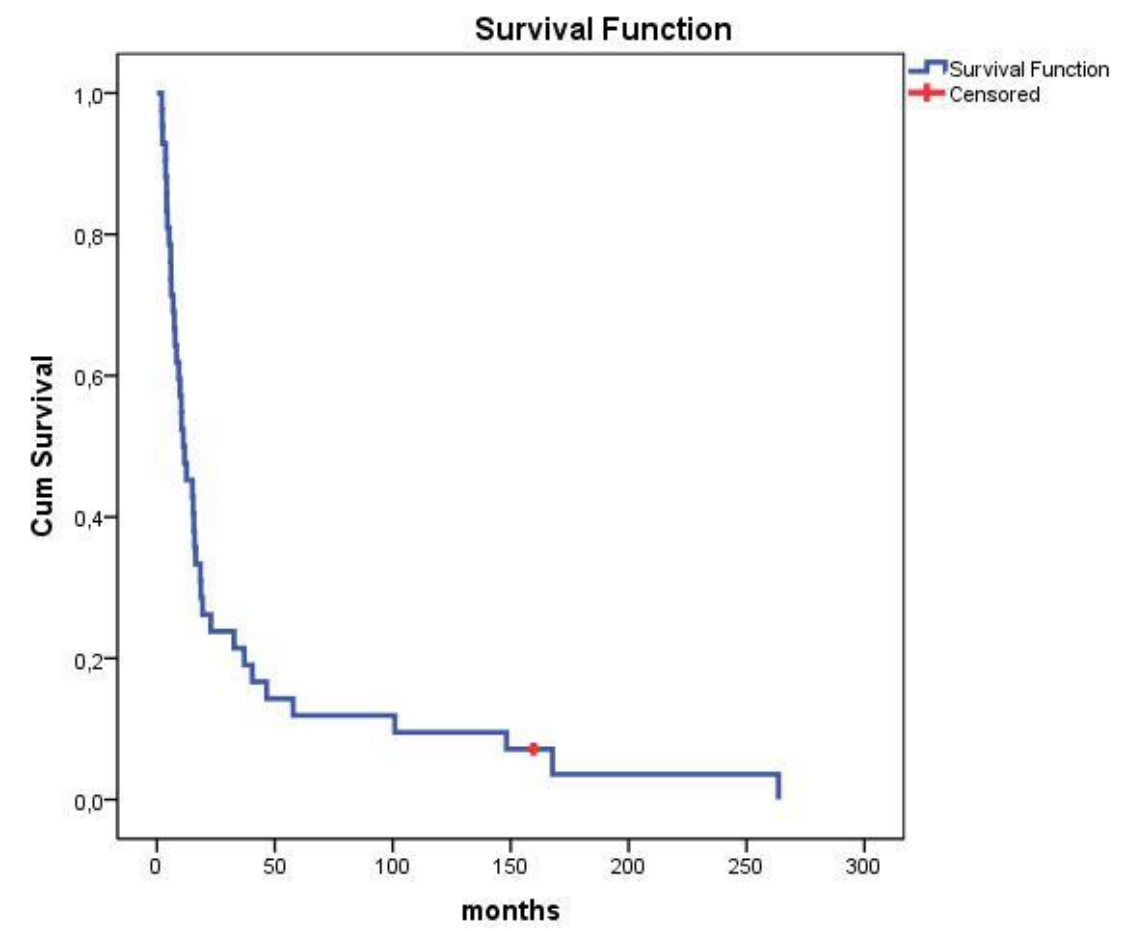

Figure 11. Total survival of the radio-chemotherapy patients independent of the clinical stage.

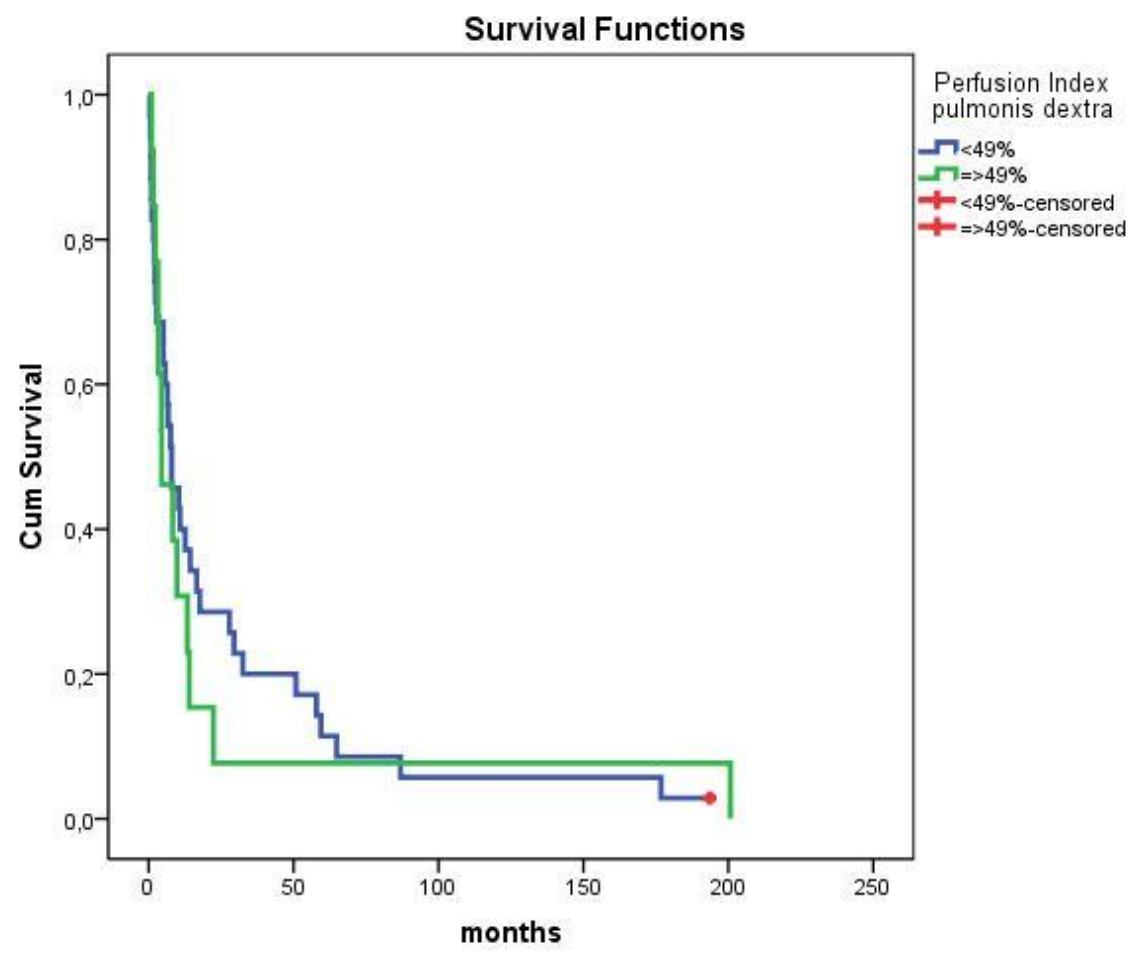

Figure 12. Survival of chemotherapy patients in months with perfusion index under and over $49 \%$ with affected right lung.

The survival of the patients treated with chemotherapy with perfusion index of left lung less than $49 \%$ had Mean=26,09 months with $\mathrm{SE}=7,02$ and $95 \%$ CI $(12,33 ; 39,84)$; Median=7,93 months with $\mathrm{SE}=1,23$ and $95 \%$
CI $(5,52 ; 10,34)$. The survival of the patients with perfusion index of the left lung more than $49 \%$ had Mean=1,03 months and 95\% CI $(1,01 ; 1,05)$, Median=1,03 months. The total survival of the patients with affected left lung 
had Mean $=25,56$ months with $\mathrm{SE}=6,89$ and 95\% CI $(12,06$; 39,07); Median=7,6 months with $\mathrm{SE}=1,48$ and $95 \%$ CI $(4,69 ; 10,51)$. The differences in survival are statistically significant $(\mathrm{P}=0,006)$ (Figure 13).

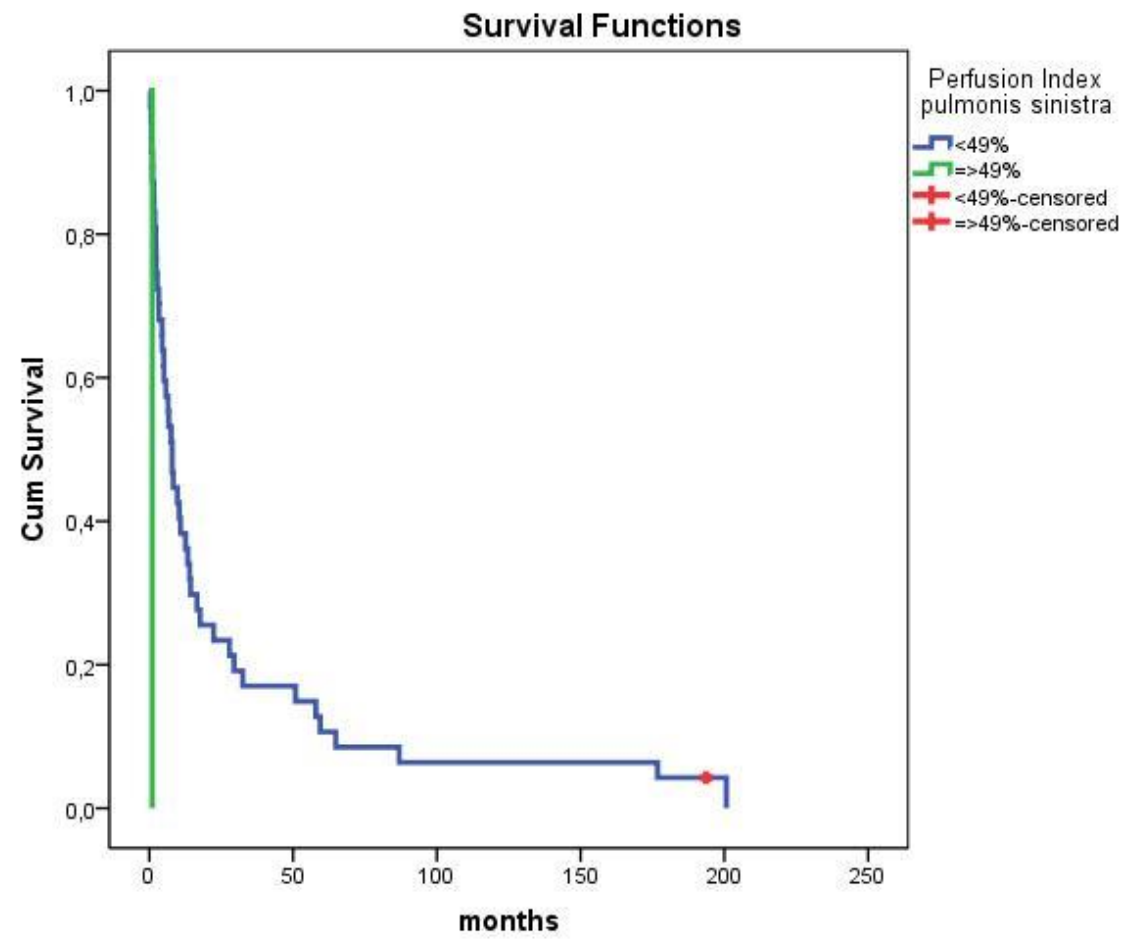

Figure 13. Survival of the chemotherapy patients in months with perfusion index over and under $49 \%$ with affected left lung.

The survival of the patients treated with chemotherapy with perfusion index of the affected lung, independent of left or right less than $49 \%$ had Mean=27,36 months with $\mathrm{SE}=7,72$ and $95 \%$ CI $(12,23 ; 42,48)$; Median $=7,93$ months with $\mathrm{SE}=2,67$ and $95 \%$ CI $(2,7 ; 13,16)$. The survival of the patients with perfusion index of affected lung more than $49 \%$ had Mean=20,72 months with $\mathrm{SE}=13,94$ and $95 \%$ CI $(0 ; 48,05)$; Median $=4,43$ months with $\mathrm{SE}=1,03$ and $95 \%$ CI $(2,41 ; 6,45)$. The total survival of the patients with affected left or right lung had Mean $=25,57$ months with $\mathrm{SE}=6,89$ and $95 \%$ CI $(12,06 ; 39,07)$; Median=7,6 months with $\mathrm{SE}=1,48$ and $95 \% \mathrm{CI}(4,69 ; 10,51)$ (Figure 14).

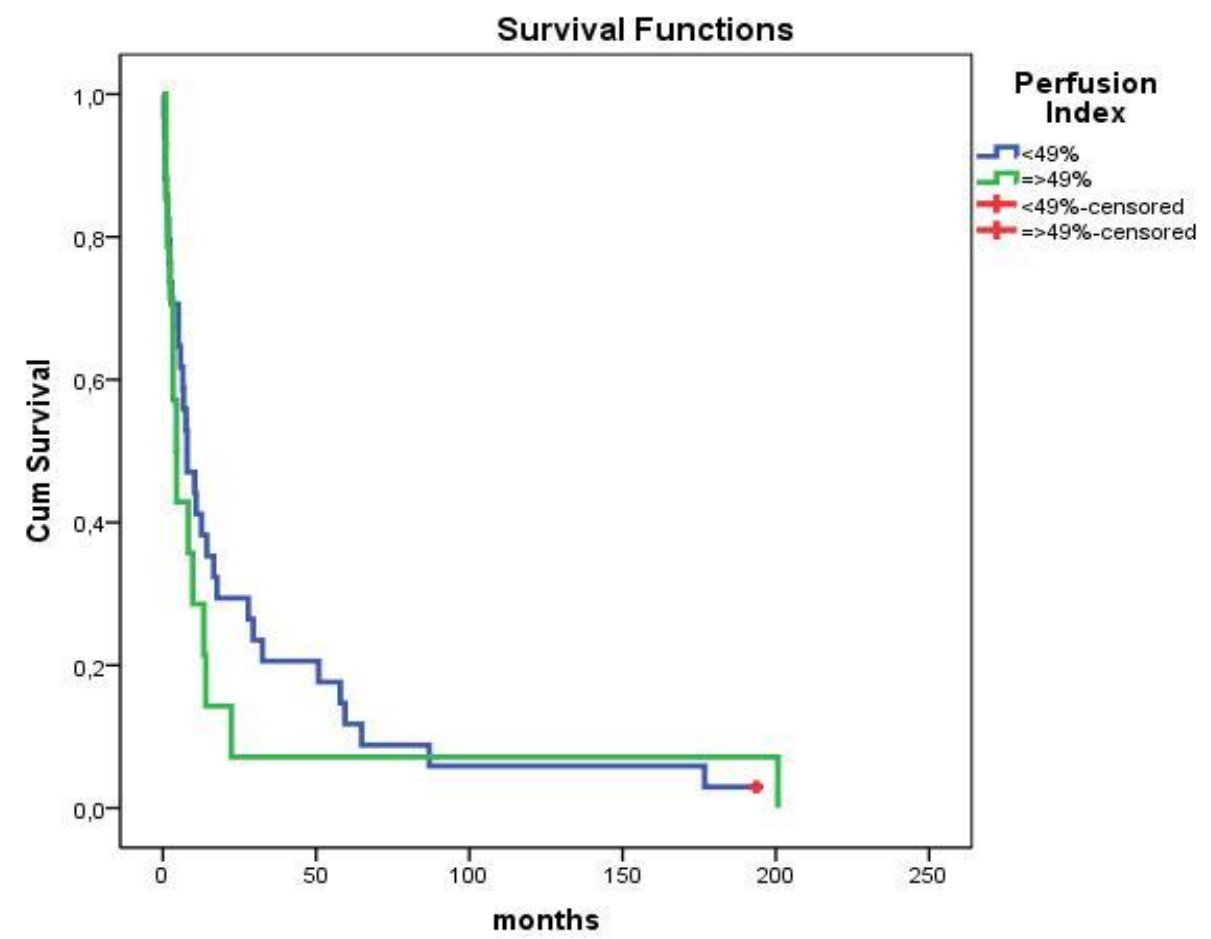

Figure 14. Survival of chemotherapy patients in months with perfusion index over and under $49 \%$, independent of the affected lung. 
The perfusion index does not appear to be statistically significant factor for the survival of the patients treated with chemotherapy $(\mathrm{P}=0,406)$. With perfusion index over $49 \%$ were only 14 patients treated with chemotherapy but half of them survived 4,4 months on average and only one patient survived around 201 months. With perfusion index less than $49 \%$ were 34 patients and half of them survived 8 months, but 2 patients survived for 177 months and one patient was still alive over 194 months. By clinical stages: the patient from stage II was with perfusion less than $49 \%$, in stages IIIA and IIIB with perfusion less than $49 \%$ were 9 , and only 4 from IIIA and 3 from IIIB accordingly with perfusion over $49 \%$, and in stage IV - with perfusion index under $49 \%$ were 15 and with perfusion index over $49 \%$ were 7 patients. In comparison with the patients treated with radio-chemotherapy the tendency for better survival at small perfusion impairments in the chemotherapy treated patients there was no better survival of the patients with small perfusion impairments. They have the opposite tendency.
GEORGIEVA N., et al.

The survival by stages for the patients treated with chemotherapy for was as follows: for stage II had Mean=7,93 months, and Median=7,93 months; for stage III A had Mean $=26,05$ months with $\mathrm{SE}=8,22$ and $95 \%$ CI $(9,95 ; 42,15)$; Median $=5,87$ months with $\mathrm{SE}=0,88$ and $95 \% \mathrm{CI}(4,16 ; 7,58)$; for stage III $\mathrm{B}$ had Mean=11,70 months with $\mathrm{SE}=4,08$ and 95\% CI $(3,71 ; 19,69)$; Median $=4,53$ months with $\mathrm{SE}=5,05$ and $95 \% \mathrm{CI}(0 ; 14,42)$; for stage IV had Mean=27,65 months with $\mathrm{SE}=9,11$ and $95 \% \operatorname{CI}(9,79 ; 45,51) ; \quad$ Median $=7,27$ months with $\mathrm{SE}=1,13$ and $95 \%$ CI $(5,06 ; 9,48)$. The total survival of the patients independent of the stage had Mean $=23,87$ months with $\mathrm{SE}=5,03$ and $95 \%$ CI $(14 ; 33,74)$; Median $=6,93$ months with $\mathrm{SE}=0,96$ and $95 \% \mathrm{CI}(5,05 ; 8,8)$ (Figure 15). The stage does not appear to be statistically significant factor for survival $(\mathrm{P}=$ $0,717)$. One reason for that could be the fact that in stage II there was only one patient and there was only one patient (from stage IIIA) and two patients from IV clinical stage have survived 201 months. Probably in part of the patients the stage has not been properly defined.

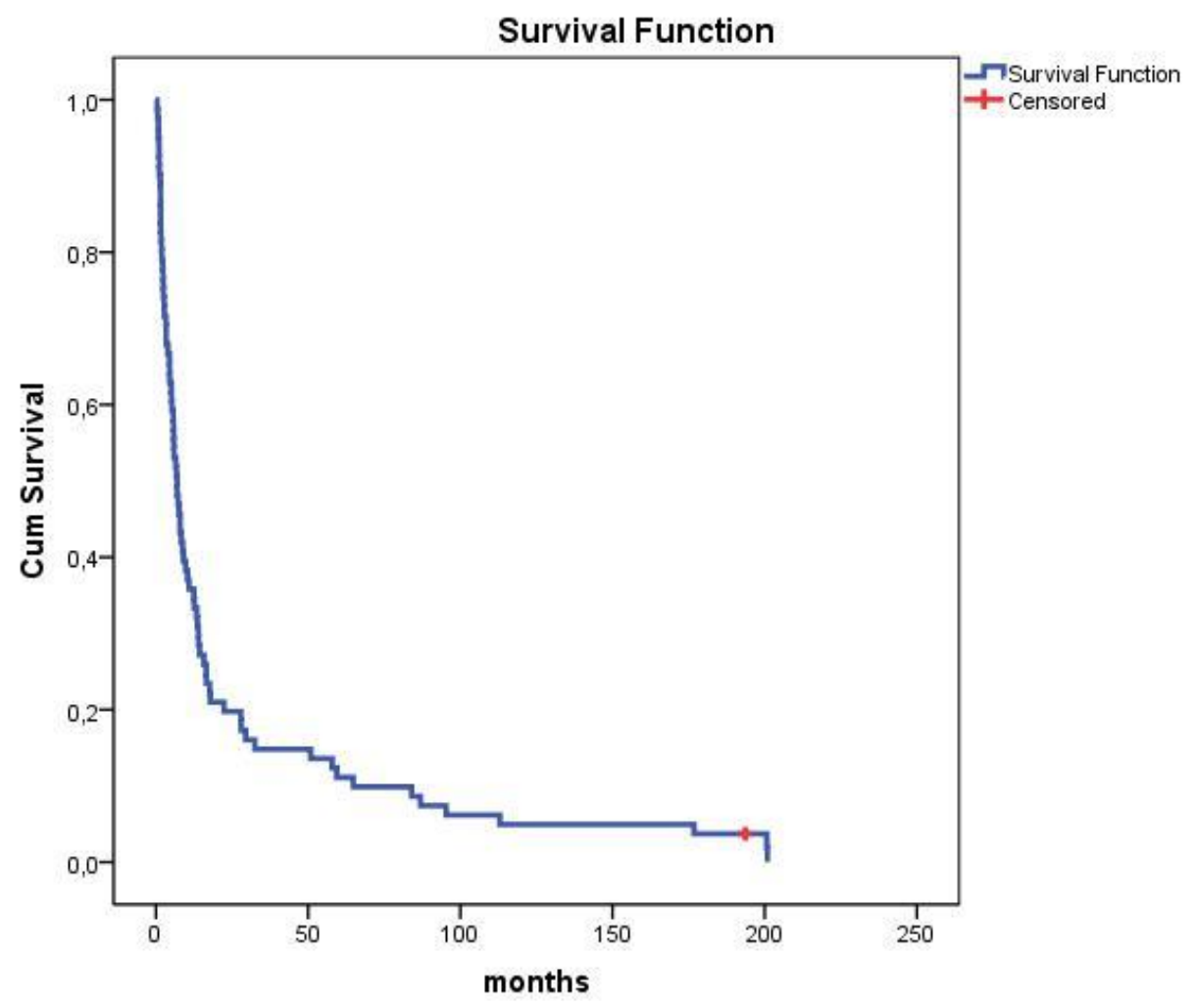

Figure 15. Total survival of all chemotherapy patients independent of the stage.

\section{DISCUSSION}

The ventilation and perfusion after radiotherapy are improved, but afterwards they slowly and progressively were worsening, which often is in relation to radiation fibrosis. In comparative assessment of all scans was established that perfusion in the affected lung was always changed. The perfusion changes could be related to pulmonary venous obstruction, invasion or compression (from the primary tumor or enlarged lymph nodes) probably with more pronounced effects on blood circulation, because the blood vessels (especially the low pressure of pulmonary veins) are more susceptible to mechanic compression than the relatively harder bronchi. The improvement of the regional perfusion and ventilation after radiotherapy is probably due 
to the shrinkage of the tumor and partial or full release of airways and pulmonary veins. Afterwards the worsening of indices of regional function is related with the progressive development of radiation fibrosis, but as this is a slow process and these patients are with brief life expectancy, probably the moment of worsening of the regional function more than before the radiotherapy would not be reached. If radiotherapy is not applied, the regional function would worsen more significantly by the dissemination of the tumor (2). There is a latent period between the radiation and the acute pulmonary reactions because of the low mitotic index of the cells in the pulmonary parenchyma. This period usually is between 1 to 3 months after the full course of radiotherapy (3). With doses over 40 Gy reduction in regional function is noticed 1 to 6 months after radiotherapy, which is not compulsory accompanied with changes in the conventional pulmonary functional tests if the affected volume is small (4). The tumor regression is associated with improvement of FEO1 and worsening of diffusion capacity after radiation. Reperfusion is not related with the improvement of the whole pulmonary (5). Radiotherapy induces dose-dependent pulmonary disfunction, which is expressed 3-6 months after radiation (5). The big fractions minimize the dosage in surrounding critical structures. The bronchial impairments and stenosis are unusual complications in conventional doses. Some chemotherapy agents are known with pulmonary toxicity and could worsen the impairments from radiotherapy (6). Lovyagin E. V. et al. (7) do not find distinct changes either in the affected parts of pulmonary parenchyma nor in non-affected parts of pulmonary parenchyma in patients with lung carcinoma and radiotherapy immediately after the treatment and in later time on perfusion scintigraphy, so the values of perfusion index stay low. The lack of pathognomonic changes in the resultant perfusion in tumors and late radiation reactions do not allow to suppose relapse.

The radiation fibrosis is result from chronic impairment of the lung. The constant changes in fibrosis are developing between 6 to 24 months, but they are often stabilized after 2 years. The patients could have X-ray image of fibrosis without history of acute pneumonitis, could be asymptomatic or to have different extent of dyspnea. Perfusion scintigraphy shows decreased perfusion in the lung after radiation. Factors which increase the risk of radiation impairments of the lung are the way of radiation, previous radiation, the usage of chemotherapy, withdrawal of steroid therapy and previous lung diseases. The pulmonary impairments are rare in partial radiation of the lung with dosage to 25-30 Gy, sometimes they appear in 30-35 Gy, they are frequent in 35-40 Gy and are usual over $40 \mathrm{~Gy}$.
GEORGIEVA N., et al. The usage of daily doses over 2,67 Gy is a significant factor, which is related to increased risk of radiation pneumonitis. Previous diseases like chronic pulmonary emphysema, pneumoconiosis, old pulmonary tuberculosis significantly increase the abilities for pulmonary fibrosis (3). In the examined patients the perfusion scintigraphy after chemotherapy and radiotherapy shows perfusion changes, which are often similar to the first scintigraphy due to the changes after radiation and in this case a relapse is difficult to define. When the changes after chemotherapy or after chemo-radiotherapy keep progressing it is a clear sign for progression of the disease. The patients with radiochemotherapy have better median of total survival and median survival by stage.

\section{CONCLUSION}

Perfusion index and stage were not statistically significant factors for the survival of patients with radio-chemotherapy and chemotherapy. Overall radio-chemotherapy treated patients have better median survival than the median survival by stage.

\section{REFERENCES}

1. Mountain CF, A New International Staging System for Lung Cancer. Chest 1986; 89: 225S-333S.

2. Fazio F., Pratt T. A., McKenzie C. G., Steiner R. E., Improvement in Regional Ventilation and Perfusion after Radiotherapy for Unresectable Carcinoma of the Bronchus. AJR 1979, 133; 191-200.

3. Movsas B., Raffin T., Epstein A. H. et al., Pulmonary Radiation Injury. Chest 1997; 111: 1061-1076.

4. Marks L. B., Spenser D. P., Bentel G. C. et al., The utility of SPECT lung perfusion scans in minimizing and assessing the physiologic consequences of thoracic irradiation. Int $J$ Radiat Oncol Biol Phys 1993; 26: 4: 659-668.

5. De Jaeger K., Seppenwoolde Y., Boersma L. J. et al., Pulmonary Function Following HighDose Radiotherapy Of Non-Small-Cell Lung Cancer. Int J Radiat Oncol Biol Phys 2003; 55: 5: 1331-1340.

6. Marks L. B., Munley M. T., Spenser D. P. et al., Quantification of radiation-induced regional lung injury with perfusion imaging. Int J Radiat Oncol Biol Phys 1997; 38:2:399409.

7. Marks L. B., Bentzen S. M., Deasy J. O et al., Radiation Dose Volume Effects in the Lung. Int J Radiat Oncol Biol Phys 2010; 76 (3 Suppl): S70-S76.

8. Lovyagin E. V., Yakovleva L. A., Muss V. F, Moerman B. A., Scintigraphic and radio bronchologic evaluation of lung cancer dissemination. Medicinskaia radiologia 1987, № 10, 20-26. (in Russian) 